\title{
BMJ Open Berkson's bias in biobank sampling in a specialised mental health care setting: a comparative cross-sectional study
}

\author{
Vincent Laliberté (iD , ${ }^{1}$ Charles-Edouard Giguère, ${ }^{2}$ Stéphane Potvin, ${ }^{2,3}$ \\ Alain Lesage, ${ }^{2,3}$ Signature Consortium
}

To cite: Laliberté V, Giguère C$\mathrm{E}$, Potvin S, et al. Berkson's bias in biobank sampling in a specialised mental health care setting: a comparative crosssectional study. BMJ Open 2020;10:e035088. doi:10.1136/ bmjopen-2019-035088

- Prepublication history for this paper is available online. To view these files, please visit the journal online (http://dx.doi org/10.1136/bmjopen-2019035088).

Received 10 November 2019 Revised 12 June 2020 Accepted 15 June 2020

Check for updates

(C) Author(s) (or their employer(s)) 2020. Re-use permitted under CC BY-NC. No commercial re-use. See rights and permissions. Published by BMJ.

${ }^{1}$ Department of Psychiatry, Jewish General Hospital, McGill University, Montreal, Quebec, Canada

${ }^{2}$ Centre de recherche de I'Institut universitaire en santé mentale de Montréal, Montréal Québec, Canada

${ }^{3}$ Département de Psychiatrie, Faculté de médecine, Université de Montréal, Montréal, Québec, Canada

Correspondence to

Dr Vincent Laliberté;

vincent.laliberte@mail.mcgill.ca

\section{ABSTRACT}

Objectives To determine whether studying aetiological pathways of depression, in particular the well-established determinant of childhood trauma, only in a specialised mental healthcare setting can yield biased estimates of the aetiological association, given that the majority of individuals are treated in primary care settings.

Design and setting Two databanks were used in this study. The Canadian Community Health Survey (CCHS) on Mental Health and Well-Being 2012 is a national survey about mental health of adult Canadians. It measured common mental disorders and utilisation of services. The Signature mental health biobank includes adults from the Island of Montreal recruited at the emergency department of a major university mental health centre. After consent, participants filled standardised psychosocial questionnaires, gave blood samples, and their clinical diagnosis was recorded. We compared the cohort of depressed individuals from CCHS and Signature in contact with specialised services with those in contact with primary care or not in treatment.

Participants There were 860 participants with depression in the CCHS and 207 participants with depression in the Signature Bank.

Primary and secondary outcomes The Childhood Experiences of Violence Questionnaire was used to measure childhood trauma in both settings. Childhood trauma is associated with depression as with other common mental and physical disorders.

Results Individuals with depression in the CCHS who reported having been hospitalised for psychiatric treatment or having seen a psychiatrist or those from Signature were found to be more strongly associated with childhood abuse than individuals with depression who were treated in primary care settings or did not seek mental healthcare in the preceding year.

Conclusions Berkson's bias limits the generalisability of aetiological associations observed in such universityhospital-based biobanks, but the problem can be remedied by broadening recruitment to primary care settings and the general population

\section{INTRODUCTION}

Berkson's bias or 'paradox' is a selection bias whereby a factor associated with a study's sampling framework gives rise to an aetiological association with the dependent variable of

\section{Strengths and limitations of this study}

The same childhood trauma brief questionnaire was used in both the population survey and the biobank.

- The study illustrates the selection bias known as Berkson's bias, where the sampling frame for case identification is associated with a different distribution of the risk factor of interest, than other larger sampling frames where the majority of cases could be found.

- The association of childhood abuse and depression found in other surveys remain valid but not as strong as ascertained only from patients in contact with specialist settings; the study points towards biobanks based on specialist settings, to also sample in primary care settings, patients identified with disorders, treated or not, to better measure the causal association and pathways.

- The ascertainment of depression was different in the populational survey (standardised questionnaires) than the biobank (admission psychiatrist).

- Childhood trauma is also in the causal pathways of comorbidities like personality disorders and substance abuse, but only the latter could be ascertained, and yet with the same limitation as measurement of depression.

interest. ${ }^{1}$ Berkson, who first pointed out this bias, identified the role of hospital sampling in the association between two conditions: cholecystitis and diabetes. ${ }^{1}$ Later, Berkson's bias was revealed in other associations, such as between respiratory disease and 'disease of the bones and organs of movement ${ }^{2}$ as well as between bladder cancer and smoking. ${ }^{3}$ In the field of psychiatry, the Berkson's bias was shown to play a role in the associations of positive and negative symptoms that we assume to constitute schizophrenia, since each of them is associated independently with mental healthcare use. ${ }^{4}$ The same would be true of the association between manic and depressive symptoms in bipolar disorder. ${ }^{5}$ Also, the Berkson's bias helped researchers show that patients with depression treated 
in specialised care settings are more strongly associated with parental loss and separation, compared with patients treated in primary care settings. ${ }^{67}$

Depression is a common chronic disease affecting $4.4 \%-5.9 \%$ of Canadian men and $11.4 \%-11.5 \%$ of Canadian women in their lifetime. ${ }^{89}$ It is associated in $60 \%$ of cases with other common chronic diseases such as diabetes, hypertension and asthma. ${ }^{10}$ While the majority of patients with depression are treated in primary care settings, ${ }^{11}$ any of the above comorbidities or comorbid behaviours increase the likelihood of receiving specialised care. Childhood abuse is a known aetiological factor in depressive disorders. Childhood trauma, such as physical and sexual abuse, has been shown to be linked to psychopathology, ${ }^{12-16}$ including depression, ${ }^{17}$ suicidal behaviours, ${ }^{15}$ substance abuse, ${ }^{16}$ personality disorders, ${ }^{18}$ physical health, ${ }^{19}$ premature mortality ${ }^{21}$ and offspring birth characteristics. ${ }^{22}$

Large demographic and biological databases are being used more and more to learn about the aetiology of illnesses, especially since the 1990s and the advent of genetic studies. ${ }^{23}$ Biobanks allow for cases and matched controls to be recruited from the same background population and social strata, and very large numbers of subjects can be used through international biobank collaborations. ${ }^{23}$ Aetiological relationships established through the use of such databanks may, however, present a bias that limits generalisability to the general population. Patients drawn from specialised care settings tend to present greater illness severity and are thus representative only of the extreme end of a disorder's spectrum. To our knowledge, although the presence of a Berkson's bias in biobanks was considered by different researchers, ${ }^{24-26}$ it was never tested empirically in any field of medicine, including psychiatry. It was against this background that we undertook a study to examine whether Berkson's bias confounded the relationship between childhood trauma and depressive disorders in a recently constituted biobank, the Signature Bank, which draws participants from among emergency room patients and subsequently hospitalised for psychiatric treatment. ${ }^{27} 28$ Their profile was compared against that of participants in Statistics Canada's Canadian Community Health Survey (CCHS) who reported depression and the use of different types of mental health services. We also sought to ascertain whether comorbid substance use disorder further influenced the strength of the association between childhood abuse and depression.

\section{METHODS}

Two different sources of data were used in this study: the CCHS and the Signature Bank.

The CCHS is a survey conducted by the Canadian government to gather health-related data in each of its provinces. Its purpose is to 'collect information on mental health status, access to and perceived need for formal and informal services and supports, functioning and disability, and covariates'. ${ }^{29}$ The 2012 CCHS covered a representative sample of about 25000 respondents aged 15 and over across the 10 provinces of Canada, almost 5000 of which lived in Quebec. ${ }^{29}$ It did not include residents in the three territories, people living on reserves and other Aboriginal settlements, full-time members of the Canadian Forces and people living in institutions. ${ }^{29}$ The survey used a cross-sectional design, and, in each province, participants were randomly selected following a three-stage sampling strategy taking into account geographical regions, household and household members. Selected households were mailed information about the survey (introductory letter and brochure). Interviewers then contacted the households by telephone to set up an appointment for an interview. The vast majority of the interviews $(87 \%)$ were conducted face to face, while the others were done over the phone. The survey was conducted by lay interviewers who administered standardised questionnaires using computer-assisted interviewing. ${ }^{29}$ The response rates (combining household and person) were $68.9 \%$ for the 2012 CCHS. ${ }^{30}$ We focused on select sociodemographic, clinical and psychosocial variables covered in the survey. The sociodemographic variables were sex (male/female) and age group (20-34, 35-49, 50-64 and $65+)$. Regarding clinical variables, we examined the presence of common mental disorders in the preceding 12 months. We focused on two diagnostic categories: major depression and substance use disorder (alcohol or other drugs). The presence of these diagnoses in the last year was ascertained during the survey using variables derived from the Composite International Diagnostic Interview. ${ }^{31}$ Substance use disorder was chosen because it was the only relevant comorbidity measured with sufficient accuracy in both the CCHS and the Signature Bank. Personality disorders were not examined because they were not adequately measured in the CCHS. The psychosocial variables investigated included service utilisation for mental health reasons in the preceding 12 months and childhood traumatic experiences. ${ }^{29}$ Service utilisation was divided into four hierarchical categories: (1) hospitalised for psychiatric treatment or saw a psychiatrist, (2) saw a family doctor but was not hospitalised or did not see a psychiatrist, (3) saw a health professional other than the ones mentioned above and (4) none of the above. In the CCHS, the presence of childhood abuse was ascertained using items from the Childhood Experiences of Violence Questionnaire, an instrument shown to be valid and reliable in assessing youth victimisation. ${ }^{32}$ Three main types of abuse were considered: physical abuse, sexual abuse and exposure to intimate partner violence. Physical abuse was further broken down into three categories: (a) slapped on face, head or ears, hit or spanked, (b) pushed, grabbed, shoved, something thrown at and (c) kicked, bit, punched, choked, burned, attacked.

The Signature Bank was created in 2010 by the Institut universitaire en santé mentale de Montréal, a mental health university institute serving a population of about half a million people in the eastern part of the Island of 
Montreal, to systematically collect biological data and 'dimensional signatures' on all patients seen at its psychiatric emergency room. ${ }^{28}$ All patients who visited the emergency room during the recruitment period that started in November 2012 were invited to participate in our study. Almost 1000 persons were initially recruited by four clinical research nurses. Sociodemographic, clinical and psychosocial data were gathered initially through standardised questionnaires. There were four data collection time points. For our purposes, we used data gathered at time point 1 in the psychiatric emergency room as participants awaited to be hospitalised, since this was the only time point where data were available for all participants. The sociodemographic data were arranged as in the CCHS. Clinical diagnoses were established by the treating psychiatrists. We selected from the Signature Bank, a cohort composed of all participants who received a depressive disorder diagnosis but were not psychotic or manic. We excluded psychosis and mania since our goal is to study common mental disorders in both specialised setting and primary healthcare. Furthermore, the CCHS was also primarily designed to report common mental disorders. The diagnoses that we accepted were major depression, chronic depression, persistent mood disorder not otherwise specified (NOS) and mood disorder NOS. The presence of a comorbid substance use disorder was determined by the treating psychiatrists as well. By definition, all participants belonged to the first CCHS hierarchical service utilisation category; that is, hospitalised for psychiatric treatment or saw a psychiatrist. Childhood trauma was assessed for the Signature Bank with the same instrument used in the CCHS; that is, the Childhood Experiences of Violence Questionnaire.

All 25113 participants who were interviewed as part of the CCHS were considered in our study (see figure 1). Participants under 20 and over 80 years of age were excluded, leaving 21506 participants. Of these, we retained only the 1162 participants who reported an episode of major depression in the preceding year. After excluding participants who reported having comorbid bipolar disorder and those who reported taking antipsychotic medication, 860 were retained for our study. All 1120 participants in the Signature Bank were considered in our study (see figure 1). Forty-seven were excluded for

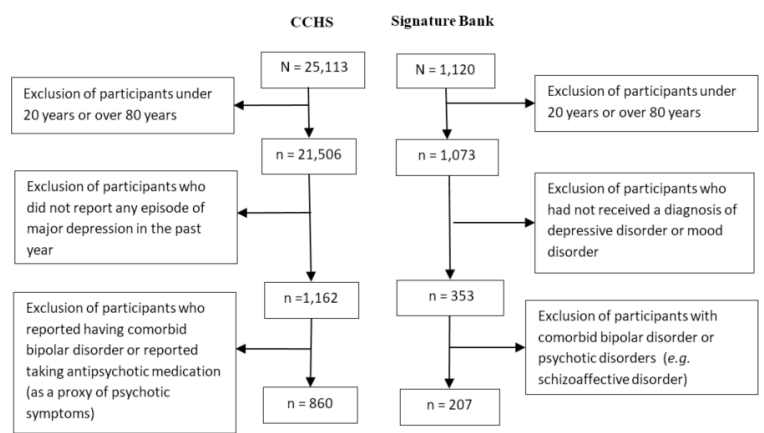

Figure 1 Selection of participants (CCHS and Signature Bank). CCHS, Canadian Community Health Survey. being under 20 years or over 80 years of age. Of the 1073 remaining, we retained only the 353 who had received a diagnosis of depressive disorder or mood disorder NOS. We were left with 207 after eliminating those with comorbid bipolar disorder or psychotic symptoms. To reference the prevalence of child abuse depending on mental health services use for people with depression, we also computed the prevalence of child abuse among people without depression in the CCHS, from all participants who were under 20 years and over 80 years, and reported it in figure 2 only.

Chi-square was used to compare the demographic characteristics of the two groups. Prevalence of childhood trauma was examined by determining and comparing the $95 \%$ CI of the prevalence of traumatic experience by level of services (see figure 2). Analyses were carried out by sex, age group and service utilisation. Logistic regressions were run for the different modes of the dependent variable, taking into account sex, ${ }^{33}$ age group, comorbid substance use disorder, ${ }^{34}$ service utilisation and group (ie, CCHS or Signature Bank). All participants in the Signature Bank consented by providing their electronic signature on a tablet, following a protocol. ${ }^{28}$ Details of the consent procedure have been published elsewhere. ${ }^{27}$

\section{Patient and public involvement}

Patients were not involved in creating this study. The results will not be disseminated to participants.

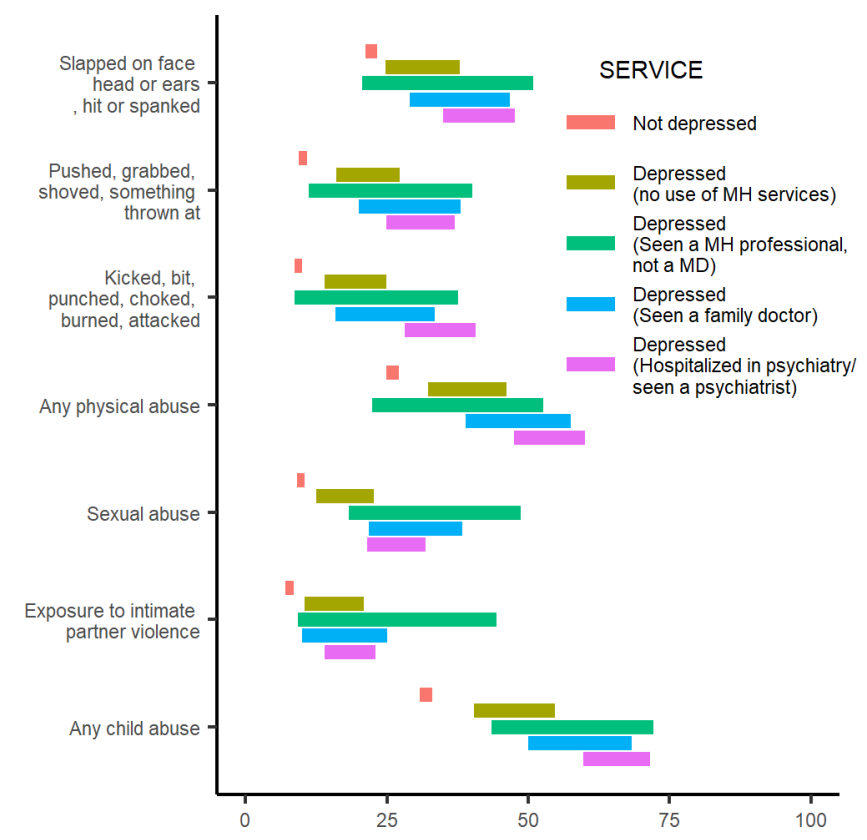

Figure $295 \% \mathrm{Cl}$ prevalence rates for each type of childhood trauma by hierarchical use of mental health services for groups of people with depression (for Canadian Community Health Survey and Signature groups combined); and, for reference, individuals without depression from the Canadian Community Health Survey. MH, Mental Health; MD, Medical Doctor. 
Table 1 Sociodemographic characteristics, clinical characteristics and service utilisation compared between CCHS and Signature Bank participants with depression

\begin{tabular}{|c|c|c|c|}
\hline & $\begin{array}{l}\text { CCHS } \\
(n=860)\end{array}$ & $\begin{array}{l}\text { Signature } \\
(n=207)\end{array}$ & $P$ values \\
\hline \multicolumn{4}{|l|}{ Sociodemographic characteristics } \\
\hline \multicolumn{4}{|l|}{ Age (in years), $\mathrm{n}(\%)$} \\
\hline 20-34 & $297(34.6)$ & $49(23.7)$ & \multirow[t]{2}{*}{0.0005} \\
\hline $65+$ & $53(06.1)$ & $13(06.3)$ & \\
\hline \multicolumn{4}{|l|}{ Clinical characteristics } \\
\hline Comorbid substance use disorder, n (\%) & $104(12.3)$ & $22(10.6)$ & 0.5466 \\
\hline \multicolumn{4}{|l|}{ Hierarchical service utilisation in the past year, $n(\%)$} \\
\hline None of the above & $337(39.2)$ & & \\
\hline
\end{tabular}

CCHS, Canadian Community Health Survey.

\section{RESULTS}

All the participants in the CCHS group reported a diagnosis of major depression, whereas all those in the Signature Bank group were diagnosed with a depressive disorder: 93\% with major depressive disorder, the rest with chronic depression, persistent mood disorder NOS and mood disorder NOS. The CCHS and Signature Bank groups are described in table 1. Proportionately, there were significantly more 20 -year to 34 -year olds in the CCHS group. However, the two groups had proportionately similar participants aged $65+$. The CCHS group also had a higher proportion of women. The proportion of participants with comorbid substance use disorder was similar in the two groups $(12.3 \%$ vs $10.6 \%, \mathrm{p}=0.54)$. Regarding hierarchical service utilisation in the preceding year, $18.9 \%$ of the participants in the CCHS group were hospitalised for psychiatric treatment or saw a psychiatrist, $31.1 \%$ saw a family doctor, $10.8 \%$ saw another professional and $39.2 \%$ used no services. All participants in the Signature Bank group were seen in an emergency room by a psychiatrist and awaited to be hospitalised for psychiatric treatment.

Figure 2 shows the results of our analysis of selfreported abuse among CCHS and Signature Bank participants by service utilisation. The 'not depressed' group corresponds to the 20344 (21 506-1162) participants in CCHS who had the appropriate age but did not have depression. They are represented in figure 2 as a reference value but not included in the multivariate analyses. In general, participants who were hospitalised for psychiatric treatment or saw a psychiatrist reported more abuse. This was especially evident when all types of abuse were considered: $65.7 \%$ (95\% CI $59.6 \%$ to $71.8 \%$ ) of participants who were hospitalised for psychiatric treatment or who saw a psychiatrist reported one form of abuse or another, compared with $49 \%$ (95\% CI $41.0 \%$ to $57.1 \%$ ) of depressed participants who used no services. Figure 2 also shows that individuals without a depressive disorder were less likely to report abuse.

The results of the logistic regression run for 'Any child abuse' are shown in table 2. Age 35-49 was found to be an independent predictor of childhood trauma, as was age 50-64, compared with age 20-34. Two other variables increased the odds of having been victimised as a child: comorbid substance use disorder and 'hospitalised for psychiatric treatment' or 'having seen a psychiatrist'. Being in the Signature Bank group also increased the odds of having suffered from 'Any child abuse' when sex, age and substance abuse were controlled. The odds were similar to those for CCHS participants with depression who saw a psychiatrist or were hospitalised for psychiatric treatment.

\section{DISCUSSION}

Our analysis demonstrates that participants with a depressive disorder in a university hospital biobank or in a community survey who are in contact with psychiatric services are more likely to have been victimised in childhood compared with the general population or with depressed individuals in the general population not in contact with specialised medical services or hospitalised for psychiatric treatment in the preceding year. Depressed individuals who were hospitalised for psychiatric treatment or saw a psychiatrist represented less than one-fifth of the individuals with depression in the 2012 CCHS population. This is in line with previous work that shows 
Table 2 List of independent predictors of the variable 'Any child abuse' from the Childhood Experience of Violence Questionnaire calculated through a regression analysis

\begin{tabular}{lccc}
\hline Effects & SE & OR & OR $95 \%$ CI \\
\hline Women (ref.) & & & \\
Men & 0.21 & 0.98 & 0.64 to 1.48 \\
\hline $20-34$ (ref.) & & & \\
\hline $35-49$ & 0.23 & 1.88 & 1.20 to 2.97 \\
50-64 & 0.22 & 1.97 & 1.28 to 3.04 \\
\hline $65+$ & 0.41 & 1.28 & 0.57 to 2.87 \\
$\begin{array}{l}\text { Comorbid substance use } \\
\text { disorder }\end{array}$ & 0.31 & 1.95 & 1.07 to 3.55 \\
\hline
\end{tabular}

No mental health services use (ref.)

\begin{tabular}{lccc}
$\begin{array}{l}\text { Saw a mental health } \\
\text { professional but not a family } \\
\text { doctor or psychiatrist }\end{array}$ & 0.34 & 1.30 & 0.66 to 2.55 \\
$\begin{array}{l}\text { Saw a family doctor but not a } \\
\text { psychiatrist }\end{array}$ & 0.27 & 1.42 & 0.83 to 2.43 \\
$\begin{array}{l}\text { Hospitalised for psychiatric } \\
\text { treatment or saw a }\end{array}$ & 0.31 & 1.93 & 1.06 to 3.53 \\
$\begin{array}{l}\text { psychiatrist } \\
\text { Signature Bank }\end{array}$ & 0.23 & 1.82 & 1.16 to 2.86 \\
\hline
\end{tabular}

ref., reference.

that only a minority of individuals with depression are seen by a psychiatrist. ${ }^{1136}$ The lower proportion of people without depression reporting abuse (figure 2) corroborates the well-established link between childhood abuse and depression. This stronger association of childhood abuse among depressed patients in contact with specialist mental health services than among depressed patients in contact only with primary care services or none at all illustrates a case of Berkson's bias in the sampling framework, given that this factor is associated both with the aetiology of the disorder and sampling framework. The bias may be explained first by the fact that patients using specialised care are more likely to present comorbidities also associated with childhood abuse, such as substance abuse $^{37}$ and personality disorders, ${ }^{38}$ as well as increased dangerosity owing, for example, to suicidal ideation. ${ }^{39}$ Our analysis confirms that when depressed individuals in both the CCHS and Signature Bank samples are considered together, comorbid substance use disorder significantly increases the likelihood of having suffered from childhood abuse. This is consistent with what has been observed in the previous research. ${ }^{34}$ A second explanation may be that childhood abuse is distributed differently by age and sex in the general population, compared with primary care or specialist settings. However, when we controlled for sociodemographic variables and comorbid substance abuse, the association was maintained, but the strength of the association between childhood trauma and depressive disorders decreased. In other words, Berkson's bias does not create a false association between childhood abuse and depression in the Signature Bank but rather reinforces it because of the sampling frame it represents.

This is the first study that provides empirical evidence of the presence of a Berkson's bias in a mental disorders biobank or any similarly constructed biobank where patients are recruited in specialist healthcare settings. The presence of this bias has implications for the aetiological links established using these biobanks for common chronic diseases (eg, diabetes, hypertension, depression) generally treated in primary care. For example, childhood physical and sexual abuses are considered to be elements of early life adversity that lead to epigenetic changes. These changes are thought to be at the root not only of depression ${ }^{28}{ }^{40}$ but also of other traits, such as impulsivity, which may underlie personality disorders and substance abuse. When genetic, physiological, psychological, functional imagery and social indicators available in the Signature biobank are explored, their mediation with childhood trauma needs to be interpreted with caution on account of the known risk of Berkson's bias inflating or deflating the association.

Two limitations of this research stem from the way data were gathered in the Signature Bank. First, it is not possible to verify the validity of the diagnoses found in patient charts. These diagnoses were not established following a structured interview but rather were formulated by an emergency room psychiatrist. However, previous psychological autopsy studies of suicide involving psychiatrists from this university hospital showed good inter-rater reliability on large categories of mental disorders from the Diagnostic and Statistical Manual of Mental Disorders, 3rd Edition (DSM-III) ${ }^{41}$ Second, the context of an emergency room itself may have a potential influence on the psychometric qualities of standardised instruments, such as the Childhood Experience of Violence Questionnaire. However, studies conducted to validate the psychometric properties of questionnaires similar to the Childhood Experience of Violence Questionnaire when used in the context of a psychiatric emergency room have demonstrated very good stability. ${ }^{27} 42$ Another potential limitation of our study stems from the fact that the Signature Bank contains a higher proportion of women relative to the CCHS sample. However, this did not affect our ability to identify Berkson's bias as the data allowed us to control for sex in the logistic regressions. Finally, our data did not allow us to take into consideration socioeconomic variables in our analysis. However, we think that such variables would not have influenced our results, since the universal coverage in Canada provides access to care to everyone. Previous work showed that individuals coming from lower economic background have not only higher rates of mood disorders but also higher rates of service utilisation (psychiatric consultation and emergency visits). ${ }^{43}$

The finding that childhood abuse was less common in the 20-34 age group in both data sets compared with the 35-49 and the 50-64 age groups is reassuring, given that 
the youngest group was most likely to remember such events at a time of heightened awareness of the consequences of child abuse, which may boost the tendency to acknowledge and report them. The data confirm that childhood abuse is on the decline, a phenomenon previously reported in Canada ${ }^{44}{ }^{45}$ and in the USA. ${ }^{46}$

The main implication of this research is that, in studying the role of childhood trauma or any other genetic or environmental aetiological factor in the development of depressive disorders, it would be worthwhile to sample not only from specialised care settings but also from the community among people in contact with primary care services or no services at all. The Signature Bank stands to benefit from broadening its sampling framework, for example, by recruiting also in family health clinics where patients with depression, whether treated or not, may also be found. In Quebec, $75 \%$ of the population see a family doctor once a year and, in $20 \%$ of these patients, the family doctor identifies or treats a mental disorder. ${ }^{47}$ Such a sampling method would afford more adequate control of Berkson's bias and bolster evidence of the strength of association with potential aetiological factors. This is why the Signature biopsychosocial bank recently started recruiting individuals from the general population as well.

Another implication is that reducing childhood trauma could be set as an intervention target in order to reduce the burden of mental disorders and their consequences. Early professional child daycare has been shown to buffer the relationship between maternal depressive symptoms and children's internalising symptoms. ${ }^{48}$ Though the matter has yet to be studied formally, early child daycare could potentially decrease childhood abuse through the development of parental skills. ${ }^{45}$ The development of daycare centres in Canada may explain the general decline in childhood abuse among the younger generation as confirmed in our study.

\section{CONCLUSION}

This study demonstrated the presence of Berkson's bias in a specialised mental healthcare biobank, a bias that has implications for attempts at drawing conclusions about the aetiological associations of psychiatric disorders. More precisely, the link between a depressive disorder and the presence of childhood abuse could be confounded by the fact that the biobank participants were recruited in a specialised care setting where patients present more comorbidity, such as substance use, and more dangerosity. When an illness treated primarily outside of hospital settings is considered, if we wish to establish aetiological links with increased generalisability, then cases must be drawn not only from specialised care settings but also from primary care health clinics. We demonstrated the presence of Berkson's bias in the Signature Bank, but the lesson should be heeded by all emerging biobanks which tend to sample cases in hospitals or other specialised care settings.
Contributors All authors participated in the study conception and design. VL and AL elaborated the specifics of the study and interpreted the data for the work. C-EG acquired the data. VL, C-EG and AL analysed it. VL drafted the work. VL, C-EG, SP and $A L$ revised it critically for important intellectual content. VL, C-EG, SP and AL worked towards final approval of the work and agreed to be accountable for all its aspects. The Signature Consortium accepted the project, supported access and analysis of data and its current head Dr Stéphane Guay approved the accepted version. AL, C-EG and SP are also members of the Signature Consortium.

Funding Vincent Laliberté is a Canadian Institute of Health Research Fellow.

Competing interests None declared.

Patient consent for publication Not required.

Ethics approval The study was approved by the research ethics board of the Centre intégré universitaire de santé et de services sociaux de l'Est-de-l'île-deMontréal (Project number 2017-809).

Provenance and peer review Not commissioned; externally peer reviewed.

Data availability statement Data are available upon reasonable request. Data can be accessed on demand through the Signature Bank online platform, by presenting a request to the database coordinator. This should be done at https://www. banquesignature.ca/en/demande-acces/procedure-de-demande-dacces/. The data obtained from this Bank for this study were already anonymised.

Open access This is an open access article distributed in accordance with the Creative Commons Attribution Non Commercial (CC BY-NC 4.0) license, which permits others to distribute, remix, adapt, build upon this work non-commercially, and license their derivative works on different terms, provided the original work is properly cited, appropriate credit is given, any changes made indicated, and the use is non-commercial. See: http://creativecommons.org/licenses/by-nc/4.0/.

ORCID iD

Vincent Laliberté http://orcid.org/0000-0003-4513-7601

\section{REFERENCES}

1 Berkson J. Limitations of the application of fourfold table analysis to hospital data. Biometrics 1946;2:47-53.

2 Sackett DL. Bias in analytic research. J Chronic Dis 1979;32:51-63.

3 Sadetzki S, Bensal D, Novikov I, et al. The limitations of using hospital controls in cancer etiology--one more example for Berkson's bias. Eur J Epidemiol 2003;18:1127-31.

4 Maric N, Myin-Germeys I, Delespaul P, et al. Is our concept of schizophrenia influenced by Berkson's bias? Soc Psychiatry Psychiatr Epidemiol 2004;39:600-5.

5 Regeer EJ, Krabbendam L, De Graaf R, et al. Berkson's bias and the mood dimensions of bipolar disorder. Int J Methods Psychiatr Res 2009;18:279-86.

6 Tennant C, Smith A, Bebbington P, et al. Parental loss in childhood: relationship to adult psychiatric impairment and contact with psychiatric services. Arch Gen Psychiatry 1981;38:1045-50.

7 Galbaud du Fort G, Newman SC, Bland RC. Psychiatric comorbidity and treatment seeking. sources of selection bias in the study of clinical populations. J Nerv Ment Dis 1993;181:467-74.

8 Murphy JM, Laird NM, Monson RR, et al. A 40-year perspective on the prevalence of depression: the Stirling County study. Arch Gen Psychiatry 2000;57:209-15.

9 Bland RC, Orn H, Newman SC. Lifetime prevalence of psychiatric disorders in Edmonton. Acta Psychiatr Scand Suppl 1988;338:24-32.

10 Schmitz N, Wang J, Malla A, et al. Joint effect of depression and chronic conditions on disability: results from a population-based study. Psychosom Med 2007;69:332-8.

11 Vasiliadis H-M, Lesage A, Adair C, et al. Do Canada and the United States differ in prevalence of depression and utilization of services? Psychiatr Serv 2007;58:63-71.

12 MacMillan HL, Fleming JE, Streiner DL, et al. Childhood abuse and lifetime psychopathology in a community sample. Am J Psychiatry 2001;158:1878-83.

13 Kessler RC, McLaughlin KA, Green JG, et al. Childhood adversities and adult psychopathology in the WHO World Mental Health surveys. Br J Psychiatry 2010;197:378-85.

14 Scott KM, Smith DR, Ellis PM. Prospectively ascertained child maltreatment and its association with DSM-IV mental disorders in young adults. Arch Gen Psychiatry 2010;67:712-9.

15 Afifi TO, MacMillan HL, Boyle M, et al. Child abuse and mental disorders in Canada. CMAJ 2014;186:E324-32. 
16 Hughes K, Bellis MA, Hardcastle KA, et al. The effect of multiple adverse childhood experiences on health: a systematic review and meta-analysis. Lancet Public Health 2017;2:e356-66.

17 Negele A, Kaufhold J, Kallenbach L, et al. Childhood trauma and its relation to chronic depression in adulthood. Depress Res Treat 2015;2015:1-11.

18 Berenz EC, Amstadter AB, Aggen SH, et al. Childhood trauma and personality disorder criterion counts: a co-twin control analysis. $J$ Abnorm Psychol 2013;122:1070-6.

19 Afifi TO, MacMillan HL, Boyle M, et al. Child abuse and physical health in adulthood. Health Rep 2016;27:10-18.

20 Brown DW, Anda RF, Tiemeier H, et al. Adverse childhood experiences and the risk of premature mortality. Am J Prev Med 2009;37:389-96.

21 Kelly-Irving M, Lepage B, Dedieu D, et al. Adverse childhood experiences and premature all-cause mortality. Eur J Epidemiol 2013;28:721-34.

22 Vågerö D, Rajaleid K. Does childhood trauma influence offspring's birth characteristics? Int J Epidemiol 2017;46:219-29.

23 Dillner J. Preface. In: Methods in biobanking. Stockholm, Sweden: Humana Press, 2011.

24 Smith SM, Nichols TE. Statistical challenges in "big data" human neuroimaging. Neuron 2018;97:263-8.

25 Jiang C, Thomas GN, Lam TH, et al. Cohort profile: the Guangzhou biobank cohort study, a Guangzhou-Hong Kong-Birmingham collaboration. Int J Epidemiol 2006;35:844-52.

26 Swanson JM. The UK Biobank and selection bias. Lancet 2012;380:110.

27 Pelletier J-F, Rowe M, François N, et al. No personalization without participation: on the active contribution of psychiatric patients to the development of a mobile application for mental health. BMC Med Inform Decis Mak 2013;13:78.

28 Lupien SJ, Sasseville M, François N, et al. The DSM5/RDoC debate on the future of mental health research: implication for studies on human stress and presentation of the signature bank. Stress 2017;20:2-18.

29 Statistic Canada. Canadian community health survey (CCHS) mental health user guide, 2013

30 Statistic Canada. The data. health reports, 2015. Available: https:// www150.statcan.gc.ca/n1/pub/82-003-x/2014009/article/14086/ data-donnee-eng.htm [Accessed 18 Apr 2020].

31 World Health Organization. Composite international diagnostic interview. Geneva, Switzerland, 1990.

32 Walsh CA, MacMillan HL, Trocmé N, et al. Measurement of victimization in adolescence: development and validation of the childhood experiences of violence questionnaire. Child Abuse Negl 2008;32:1037-57.
33 Wolfe VV. Child sexual abuse. In: Mash EJ, Barkley EJ, ed. Assessment of childhood disorders. 4 edn. New York: Guilford Press, 2007: 685-748.

34 Khoury L, Tang YL, Bradley B, et al. Substance use, childhood traumatic experience, and posttraumatic stress disorder in an urban civilian population. Depress Anxiety 2010;27:1077-86.

35 Tonmyr L, Shields M. Childhood sexual abuse and substance abuse: a gender paradox? Child Abuse Negl 2017;63:284-94.

36 Kessler RC, Merikangas KR, Wang PS. Prevalence, comorbidity, and service utilization for mood disorders in the United States at the beginning of the twenty-first century. Annu Rev Clin Psychol 2007;3:137-58.

37 Swadi H, Bobier C. Substance use disorder comorbidity among inpatient youths with psychiatric disorder. Aust N Z J Psychiatry 2003;37:294-8.

38 Zimmerman M, Chelminski I, Young D. The frequency of personality disorders in psychiatric patients. Psychiatr Clin North Am 2008;31:405-20.

39 Hirschfeld RM, Russell JM. Assessment and treatment of suicidal patients. N Engl J Med 1997;337:910-5.

40 Turecki G, Brent DA. Suicide and suicidal behaviour. Lancet 2016;387:1227-39.

41 Kim C, Lesage A, Seguin M, et al. Patterns of co-morbidity in male suicide completers. Psychol Med 2003;33:1299-309.

42 Giguère Charles-Édouard, Potvin S, Signature Consortium. The drug abuse screening test preserves its excellent psychometric properties in psychiatric patients evaluated in an emergency setting. Addict Behav 2017;64:165-70.

43 Vanasse A, Courteau J, Lesage A, et al. [Health inequities in mood disorders based on material and social deprivation in dwelling sectors ]. Can J Psychiatry 2012;57:772-81.

44 Collin-Vézina D, Hélie S, Trocmé N. Is child sexual abuse declining in Canada? an analysis of child welfare data. Child Abuse Negl 2010;34:807-12.

45 Ligier F, Giguère C-E, Séguin M, et al. Survey evidence of the decline in child abuse in younger Canadian cohorts. Eur J Pediatr 2019;178:1423-32

46 Finkelhor D, Turner $\mathrm{H}$, Ormrod $\mathrm{R}$, et al. Trends in childhood violence and abuse exposure: evidence from 2 national surveys. Arch Pediatr Adolesc Med 2010;164:238-42.

47 Ouadahi Y, Lesage A, Rodrigue J, et al. Are mental health problems detected by the general practitioners? perspective of general practitioners according to the administrative database. Sante Ment Que 2009;34:161-72.

48 Herba CM, Tremblay RE, Boivin M, et al. Maternal depressive symptoms and children's emotional problems: can early child care help children of depressed mothers? JAMA Psychiatry 2013;70:830-8. 